Politik erklären. Hier wird deutlich, welchen beschränkten Erklärungswert ein Begriff wie ,Autochthonie' hat, wenn er so eng gefaßt wird wie in dieser Untersuchung. Yash P. Ghai hat an anderer Stelle ${ }^{3}$ diese Begriffsbildung kritisiert, die die Erörterung von vornherein auf die rechtstechnische Unterbrechung der verfassungsrechtlichen Kontinuitätskette begrenzt und materielle Entwicklungen des Verfassungsrechts der neuen Staaten ausklammert. So will es z. B. nicht einleuchten, daß Tansanias Verfassung nur „auf niederer Stufe autochthon“ ist (S. 110), obwohl dieser Staat sich wohl wie kaum ein anderes Commonwealth-Mitglied in seiner internen Struktur vom ,Westminster export model' entfernt und sein Verfassungsrecht damit in einem weiteren Sinne, autochthonisiert' hat. Hier wird deutlich, daß der Abbruch der Kontinuitätskette nur ein denkbares - vielleicht gar nicht das wirksamste - Mittel zur Autochthonisierung der Rechtsordnung ist. Sicherlich lag eine solche Autochthonie im weiteren Sinne außerhalb des Rahmens, den der Verfasser für seine hier besprochene Untersuchung gesteckt hatte. Es bleibt jedoch zu hoffen, daß die angedeutete Problematik bald zum Gegenstand einer eigenen Analyse gemacht wird, um einen umfassenden Überblick über die rechtlichen Aspekte und Konsequenzen der britischen Dekolonisationspolitik zu vermitteln. Der Verfasser hat mit seinem Buch einen recht speziellen, darum aber nicht weniger wertvollen Beitrag zur Beschreibung der juristischen Entkolonialisierung im Commonwealth geleistet.

Alexander Dix

\title{
KI-Whan NAM
}

Völkerrechtliche und staatsrechtliche Probleme des zweigeteilten Korea und die Frage der Vereinigung der koreanischen Nation

Europäische Hochschulschriften - Rechtswissenschaft Bd. 113, Herbert Lang, Bern und Peter Lang, Frankfurt/M., 1975, 239 S., sFr. 43, 20

Es war am 4. Juli 1972, als nicht nur Koreaner, sondern viele andere Völker in der Welt das sogenannte „Nord- und Südabkommen“ begeistert aufnahmen. In diesem Abkommen vereinbarte Korea die Wiedervereinigung des Landes durch „unabhängige koreanische Bestrebungen auf friedlichem Wege und auf der Grundlage des ursprünglich homogenen Volkes". Aber die koreanische Bevölkerung ist von der gegenwärtigen feindseligen politischen Entwicklung zwischen Nord- und Südkorea so enttäuscht, daß sie das „wirkliche Ziel“, den koreanischen Dialog, seit 1971 anzweifelt ${ }^{1}$. Deshalb untersuchen z. Z. nicht nur $\mathrm{ich}^{2}$, sondern viele Wissenschaftler, wie z. B. Gregory Henderson, Harry Sichrovsky und Joungwon A. $\mathrm{Kim}^{3}$, den Grund des „erfolglosen“ koreanischen Dialoges und die Möglichkeit der Wiedervereinigung Koreas. In diesem Sinne gratuliere ich Herrn Ki-Whan Nam für seine Promotion mit dieser Dissertation herzlich.

Das erste Kapitel (S. 8-61) handelt über die völkerrechtliche Anerkennung des Staates. Als theoretischen Teil faßt der Verfasser die Voraussetzungen der Pflicht

3 ICLQ 1973, S. 403 ff., 414 f.

1 Vgl. Youn-Soo Kim: Korea - Die staatsrechtliche Problematik eines geteilten Staates im Vergleich zur Bundesrepublik Deutschland. In: VRƯ, 4. Heft 1974, S. 427-441

2 Youn-Soo Kim: Die Teilung und Wiedervereinigung Koreas 1945-1973 - Analyse und Dokumente, Kiel 1973

3 Gregory Henderson: Divided Nation in a Divided World. New York 1974; Harry Sichrovsky: Korea Report - Vom Bruderkrieg zur Wiedervereinigung? Wien 1973; Joungwon A. Kim: Divided Korea The Politics of Development 1945-1972. Cambridge/Massachusetts 1975 
zum Recht und die Formen der Anerkennung sowie die Wirkung der vorläufigen (de facto) und endgültigen (de jure) Anerkennung des Staates zusammen. Das zweite Kapitel (S. 62-97) behandelt die internationale Stellung Koreas bis 1948: Korea war von Japan „kriegsmäßig besetzt, und zwar unter Bruch des allgemeinen Völkerrechts und einer ganzen Anzahl von Japan unterzeichneter Verträge" (S. 81-82). Erst der japanische Überfall auf das amerikanische Pearl Harbor löste den Krieg und damit den effektiven bewaffneten Widerstand gegen die andauernden japanischen Rechtsbrüche aus (S. 85). Mit der Beseitigung der japanischen Okkupation im Jahr 1945 hatte Korea seine völkerrechtliche Handlungsfreiheit und damit auch die Fähigkeit zur Ausübung seiner Gebietshoheit wiedererlangt. Allerdings wurde die völkerrechtliche Handlungsfähigkeit Koreas sofort wieder durch die Besetzung des Landes durch die zwei Großmächte, UdSSR und USA, beschränkt (S. 94).

Im dritten Kapitel (S. 98-172) behandelt Herr Nam die Frage der gegenwärtigen Rechtslage Koreas: Wie kann z. B. das faktische Nebeneinanderbestehen zweier koreanischer Staatsgebilde in Korea juristisch erklärt werden? Der Verfasser stellt fest, daß die Koreanische Demokratische Volksrepublik (KDVR) die These der „Zwei souveränen Staaten" hat (S. 100-101 und 169), während die Republik von Korea (RvK) sich auf "the only state of the entire Korean nation“, die „Bürgerkriegstheorie“ (S. 102 und 170) beruft. Diese „Behauptung“ bedarf aber notwendig einer Korrektur. Herr Nam schrieb u. a.: „Die RvK weigert sich, die KDVR als Staat anzuerkennen (S. 103). Aber der Verfasser "muß auch wissen“, daß die KDVR auch Südkorea als Staat nicht anerkennt. Die beiden koreanischen „Halb-Staaten“ haben vereinbart, das von dem Verfasser oft zitierte "gemeinsame Kommuniqué vom 4. Juli 1972“ (S. 114 und 120) nicht als „nord- und südkoreanisches Abkommen “, sondern als „Nord- und Südabkommen für die friedliche Wiedervereinigung des Landes“ zu bezeichnen, weil Korea, d. h. KDVR und RvK, nur „two governments and one nation" anerkennen kann".

Der Verfasser gab den nordkoreanisch-sowjetischen Vertrag über die kulturelle Zusammenarbeit von 1956 und den sowjetischen Antrag für die beiden koreanischen Beitritte in der UNO von 1955 als „Beweis“ für die nordkoreanische „These der zwei souveränen Staaten" an (S. 100-101). Aber solche Verträge zwischen den koreanischen „Halb-Staaten“ und ihren „Schutzmächten“ wurden nicht nur von Nordkorea, sondern auch von Südkorea mit den USA abgeschlossen, während der nordkoreanisch-sowjetische Vertrag mit keinem Wort über „zwei koreanische Staaten" spricht. Es war eine Tatsache, daß die UdSSR solchen Antrag an die UNO nicht nur im Jahr 1955, sondern auch 1957 stellten. Aber der Verfasser „verschweigt", daß die amerikanische Politik für die nord- und südkoreanische gemeinsame Mitgliedschaft in der UNO wegen des Widerstandes der UdSSR am 30. Juli 1975 gescheitert war ${ }^{5}$.

Insbesondere die nordkoreanische Außenpolitik selbst spricht gegen die „These der zwei Staaten“, wie z. B.

4 Vgl. Interview Li Hu-Raks am 5. Juli 1972 in allen koreanischen Zeitungen vom selben Tag und auch

Hankook-Ilbo, 27. Juni 1973 .
5 Vgl. Hak-Joon Kim: Hankook-Munche-wa-Kukche-Chongch'i (Die Frage Koreas und Internationale Politik). Seoul 1975, S. $146-164$ 
1. Kim Il-Sung lehnte aus Protest gegen die sowjetische „Zwei-Korea“-Politik ab, an den Internationalen Universitätssportfestspielen 1973 in Moskau teilzunehmen, als die UdSSR auch Südkorea einlud'.

2. Kim Il-Sung lehnte den südkoreanischen Vertrag für die gemeinsame Mitgliedschaft in der UNO mit Nordkorea ab, weil er die Teilung des Landes verewigt. Dagegen fordert Kim Il-Sung die Gründung der Konföderalen Republik Koryo aus beiden Korea, und zwar bis zur völligen Wiedervereinigung, weil Nordkorea nicht „zwei Staaten“, sondern nur „zwei Regierungen“ auf der koreanischen Halbinsel sieht ${ }^{7}$.

Es war ein „Glück im Unglück“, daß das „kleine“ Mißverständnis des Verfassers keine „große“ Rolle für die Schlußfolgerung des dritten Kapitels gespielt hat. Herr Nam stellt fest (S. 169):

Seit der Bildung zweier koreanischer Republiken in Korea ist die Entwicklung der Lage so weit fortgeschritten, daß keine der beiden Republiken die andere einfach weiterhin ignorieren oder gar gänzlich verdrängen kann. Man wird deshalb jede Entwicklung begrüßen, die es verhindert, daß sich die beiden koreanischen Republiken im Verhältnis zueinander zu zwei souveränen Staaten fortentwickeln.

Um eine solche Entwicklung zu vermeiden, erkennen die beiden „Regierungen auf der koreanischen Halbinsel" sich gegenseitig nicht als Staaten an.

Das vierte Kapitel (S. 173-217) ist der wichtigste Teil und untersucht, welche Konsequenzen sich aus der gegenwärtigen Rechtslage Koreas für die Frage der Wiedervereinigung Koreas ergeben. Dafür gibt es nach der Untersuchung von Herrn Nam zwei Möglichkeiten, und zwar die Auffassung der Zweistaatenthese und die Identitätslehre. Aber bei beiden Theorien gibt es Schwierigkeiten. Bei der Auffassung der Zweistaatenthese (S. 174-186) konnte es z. B. eine politische Gefahr für die Einheit Koreas geben. Sobald die KDVR ausdrücklich Staatsqualität zugesteht, unterscheidet sich das Verhältnis der KDVR zur RvK in keiner Weise von dem Verhältnis der KDVR zu anderen Staaten. Damit würden der koreanischen Politik der friedlichen Wiedervereinigung auf der Grundlage des Selbstbestimmungsrechts völkerrechtliche Hindernisse in den Weg gelegt.

Auch die Identitätslehre (S. 187-207) enthält eine politische Gefahr, „wenn sie die RvK als einzigen koreanischen Staat auf koreanischem Boden und damit als identisch mit dem koreanischen Gesamtstaat ansieht", weil man damit „ungewollt alle Voraussetzungen für eine eigene Staatlichkeit der KDVR schafft, was letztlich zur Annahme der vollzogenen Teilung Koreas führen muß, deren innere Konsequenz die künftige Anerkennung auch der KDVR als Völkerrechtssubjekt sein würde".

Politisch zweckmäßig kann, nach der Meinung des Verfassers, „daher nur ein Kompromiß, ein Ausgleich beider Konzeptionen“ sein, nämlich „die modifizierte Zweistaatenthese“ (S. 217). Es gibt zwei Möglichkeiten für die Wiedervereinigung Koreas:

1. im Wege des freiwilligen Anschlusses einer koreanischen Republik an die andere, oder

\footnotetext{
6 Korean Central News Agency (KDVR), 19. August 1973

7 Vgl. Speech of the respected and beloved leader Comrade Kim Il-Sung at Pyongyang Massmeeting held today in Honour of the Party and Government Delegation of the Czechoslovakia Socialist Republic. In: Korean Daily News (Tokio), 26. Juni 1973, S. 26-36
} 
2. durch den Zusammenschluß beider koreanischen Republiken (S. 213).

Während sich auf der Basis der Zweistaatenlehre - in erster Linie - der Weg des Zusammenschlusses anbietet, ist mit der Identitätstheorie nur die Form des Anschlusses vereinbar. $\mathrm{Da}$ aber die Theorie des Anschlusses nur eine „rein theoretische Bedeutung besitzt", bleibt daher praktisch nur der Weg des Zusammenschlusses, ein Weg, der rechtlich und logisch nicht unter Außerachtlassung der modifizierten Zweistaatenthese beschritten werden kann (S. 217).

Man muß hier die wissenschaftliche Bedeutung dieser Dissertation „hoch schätzen“, weil sie eine der ersten Arbeiten ist, die die Frage Koreas rechtswissenschaftlich untersucht hat. Diese Untersuchung „beweist“ deutlich, daß Korea nur auf dem friedlichen Wege wiedervereinigt werden kann, was Korea nie vergessen soll.

Youn-Soo Kim 